\title{
Angiotensin-converting enzyme 2 attenuates the metastasis of non-small cell lung cancer through inhibition of epithelial-mesenchymal transition
}

\author{
YAN-RONG QIAN*, YI GUO*, HUAN-YING WAN, LIANG FAN, YUN FENG, \\ LEI NI, YI XIANG and QING-YUN LI
}

Respiratory Department, Ruijin Hospital, Shanghai Jiaotong University School of Medicine, Shanghai 200025, P.R. China

Received January 9, 2013; Accepted March 8, 2013

DOI: $10.3892 / o r .2013 .2370$

\begin{abstract}
Angiotensin-converting enzyme 2 (ACE2) is a key enzyme of the renin-angiotensin system (RAS). ACE2 plays a critical counterbalancing role by degrading angiotensin II (Ang II) to Ang 1-7. Recent studies suggest that RAS influences tumor growth and development by its paracrine effects on the tumor microenvironment. Epithelial-mesenchymal transition (EMT) is now thought to be a process that plays a fundamental role in tumor progression and metastasis. In the present study, we investigated the role of ACE2 in lung cancer metastasis and the mechanism of EMT. This is the first study to elucidate the mechanism through which the overexpression of ACE2 in the A549 lung cancer cell line decreases metastasis formation in vivo and upregulates the expression of E-cadherin both in vitro and in vivo. We also observed the downregulation of vimentin, which supports a role of ACE2 in influencing EMT in lung cancer. Further analysis indicated that ACE2 abrogated the upregulation of TGF- $\beta 1$-induced EMT markers, such as vimentin and $\alpha$-smooth muscle actin ( $\alpha$ SMA) in vitro in A549 cells. Finally, exposing A549 cells stably expressing ACE2 to DX600, an inhibitor of ACE2, recovered the sensitivity of lung cancer cells to TGF- $\beta 1$-mediated induction of EMT. Our study demonstrated that ACE2 attenuated the metastasis of lung cancer and may serve as a target for new strategies to inhibit EMT in cancer cells.
\end{abstract}

\section{Introduction}

Lung cancer continues to be the leading cause of death due to cancer in Western countries. In 2012, lung cancer was esti-

Correspondence to: Professor Huan-Ying Wan, Respiratory Department, Ruijin Hospital, Shanghai Jiaotong University School of Medicine, Ruijin 2nd Road, Shanghai 200025, P.R. China

E-mail: spriteqian@163.com

*Contributed equally

Key words: lung cancer, metastasis, epithelial mesenchymal-transition, E-cadherin mated to account for approximately $26 \%$ of female and $29 \%$ of male deaths due to cancer (1). Among them, the formation of metastasis is the most common cause of death.

The process of epithelial-mesenchymal transition (EMT) plays a fundamental role in tumor progression and formation of metastasis. In EMT, epithelial tumor cells with a cobblestone phenotype acquire mesenchymal cell characteristics with a spindle/fibroblast-like morphology (2). This process involves the loss or downregulation of epithelial markers, including E-cadherin and upregulation of mesenchymal molecular markers such as vimentin and $\alpha$-smooth muscle actin ( $\alpha$ SMA). During the acquisition of EMT, loss of epithelial markers, especially E-cadherin, is a critical process that is regulated by several important transcriptional repressors. The processes of EMT may be triggered by many growth factors, including transforming growth factor $\beta 1$ (TGF- $\beta 1$ ), which is the most important stimulus that can be influenced by the tumor microenvironment.

In recent years, the role of the renin-angiotensin system (RAS) in tumor progression or metastasis has been extensively characterized (3-8). Changes in the expression of RAS components, particularly in local tumor tissue, appear to correlate with tumor grade and clinical outcome (9). We previously reported that the low expression of angiotensin-converting enzyme 2 (ACE2) is associated with tumor grade in lung cancer (10). Lower levels of ACE2 expression co-localized with areas of malignant tumors. Moreover, recent studies suggest that angiotensin II (Ang II), which is the key effector of the RAS system, was found to be enhanced in the EMT process of intrahepatic cholangiocarcinoma at a local tissue level (11). It is of note that marked progress continues to be made in unraveling the contribution of the RAS system, particularly the role of the vasodepressor pathway composed by ACE2.

As we previously reported, decreased local expression of ACE2 was shown to correlate with the progression of lung cancer while transcriptional overexpression of ACE2, using an adenoviral-mediated plasmid, reduced invasion and angiogenesis in A549 cells in vitro and inhibited tumor growth in a mouse model (10). In our present study, we sought to investigate whether ACE2 inhibits metastasis in lung cancer and whether it influences the EMT process in lung cancer cells using the A549 cell line as a model. 


\section{Materials and methods}

Materials and reagents. F-12K nutrient mixture (F-12K) and fetal bovine serum (FBS) were obtained from Gibco (Grand Island, NY, USA). Antibiotics (100 U/ml penicillin and $100 \mathrm{mg} / \mathrm{ml}$ streptomycin) were purchased from Invitrogen Life Technologies (Carlsbad, CA, USA). Recombinant human TGF- $\beta 1$ was purchased from R\&D Systems (Minneapolis, MN, USA). Mouse anti- $\beta$-actin antibody was from Santa Cruz Biotechnology Inc. (Santa Cruz, CA, USA). Mouse monoclonal anti-E-cadherin and mouse monoclonal anti-vimentin were from BD Biosciences (San Diego, CA, USA). Goat polyclonal antibody to ACE2 was from R\&D Systems. Rabbit polyclonal antibody to $\alpha$ SMA was from Abcam (Cambridge, MA, USA). DX600 was from AnaSpec (San Jose, CA, USA).

Cell lines and culture conditions. A549 cells were purchased from the Cytology Center of the Chinese Academy of Sciences (Shanghai, China). Cells were maintained in F-12K nutrient mixture (F-12K; Gibco, Carlsbad, CA, USA) containing 10\% FBS and $100 \mathrm{U} / \mathrm{ml}$ each of penicillin/streptomycin at $37^{\circ} \mathrm{C}$ in $5 \% \mathrm{CO}_{2}$. The media were replaced every $48 \mathrm{~h}$. In order to examine cell survival, cells were cultured up to $70 \%$ confluence and serum-starved overnight. They were then treated with TGF- $\beta 1$ in culture medium containing $1 \%$ FBS for $72 \mathrm{~h}$. DX600, which is a specific inhibitor of ACE2, was added to the culture $30 \mathrm{~min}$ before the addition of TGF- $\beta 1$.

Gene transfection. To analyze the function of ACE2, we established stable clones of A549 cells overexpressing ACE2 (A549-ACE2). The transfection procedure was performed as previously reported (12). Briefly, infection of A549 cells with MSCV-ACE2 resulted in robust ACE2 expression compared with A549 cells infected with the vector alone. Overexpression of ACE2 was confirmed by western blot analysis as described below.

$R T-P C R$. The mRNA levels of Twist, Snail2, ZEB1 and E-cadherin were examined by RT-PCR. The procedure of RT-PCR was performed as previously reported (13). Total RNA was extracted from cells or tumor tissues using TRIzol reagent (Invitrogen, Karlsruhe, Germany) according to the manufacturer's protocol. To determine the mRNA transcript level from cDNA, PCR was carried out using the following primers: E-cadherin forward, 5'-CCACCAAAGTCACGCTGAAT AC-3' and reverse, 5'-GGAGTTGGGAAATGTGAGCAA-3'; Twist forward, 5'-TCTCGGTCTGGAGGATGGAG-3' and reverse, 5'-GTTATCCAGCTCCAGAGTCT-3'; Snail2 forward, 5'-GAGCATTTGCAGACAGGTCA-3' and reverse, 5'-CCTCA TGTTTGTGCAGGAGA-3'; ZEB1 forward, 5'-GCACAACC AAGTGCAGAAGA-3' and reverse, 5'-GCCTGGTTCAGGA GAAGATG-3'; and $\beta$-actin, which served as an internal control, forward, 5'-AAGATGACGCAGATCATGTTTGAG-3' and reverse, 5'-AGGAGGAGCAATGATCTTGATCTT-3'.

SDS-PAGE and western blot analysis. Cultured A549 cells $\left(10^{6}-10^{7}\right)$ were washed with cold phosphate-buffered saline (PBS; Mediatech) three times and disrupted in $0.2 \mathrm{ml}$ ice-cold cell lysis buffer containing 10\% phenylmethanesulfonyl fluoride (PMSF). After incubation for $5 \mathrm{~min}$ at room temperature, cells were scraped from the 6-well plates. Total cell lysates were sonicated, and insoluble materials were removed by centrifugation at $13,000 \mathrm{rpm}$ for $15 \mathrm{~min}$ at $4^{\circ} \mathrm{C}$. Protein concentrations were determined by the Bradford method (Bio-Rad, Herts, UK). Equal amounts of protein $(10 \mu \mathrm{g})$ were separated on an $8 \%$ SDS-polyacrylamide gel (Bio-Rad). After electrophoresis, separated proteins were transferred onto immunoblot polyvinylidene difluoride (PVDF) membranes (Merck Millipore, USA). Membranes were then blocked with 5\% non-fat dried milk in Tris-buffered saline with Tween-20 (TBST) for $1 \mathrm{~h}$ at room temperature. Primary antibodies were added to TBST, and membranes were incubated overnight at $4^{\circ} \mathrm{C}$ on a rocking platform. Primary antibodies used were; mouse anti- $\beta$-actin $(1: 3,000)$ from Santa Cruz Biotechnology Inc., mouse monoclonal anti-E-cadherin $(1: 1,500)$ and mouse anti-vimentin $(1: 1,500)$ from BD Biosciences. Rabbit polyclonal to $\alpha$ SMA (1:500) was from Abcam. After three washing steps with TBST (15 min each), membranes were probed with the corresponding anti-rabbit horseradish peroxidase (HRP)conjugated secondary antibody for $2 \mathrm{~h}$ at room temperature. Membranes went to a second stage of washing in TBST, three times each for $15 \mathrm{~min}$. Immunoblots were visualized by enhanced chemiluminescence (GE Healthcare, Chalfont St. Giles, UK). $\beta$-actin band density was used as a loading control, and results were digitalized and quantified using ImageJ software.

Animal experiments. Six-week-old male athymic nu/nu mice were purchased from the Shanghai Laboratory Animal Center of Chinese Academy of Sciences (Shanghai, China) and maintained under specific pathogen-free conditions. Animals were maintained in a temperature-controlled room (at $22^{\circ} \mathrm{C}$ ) and supplied with food and water. A single-cell suspension containing $2 \times 10^{6}$ cells in $0.1 \mathrm{ml}$ phosphate-buffered saline was injected into the lateral tail veins of nu/nu mice. There were six mice per group. Three weeks after treatment, mice were sacrificed and then lung, liver and brain tissues were extracted, fixed with $4 \%$ formaldehyde, and the number of metastatic colonies was counted under a dissecting microscope. For the lung cancer xenograft model, wild-type or ACE2 overexpressing A549 cells $\left(2 \times 10^{7}\right.$ in $0.1 \mathrm{ml}$ PBS $)$ were transplanted subcutaneously. Four weeks later, mice were sacrificed, and tumor tissues were harvested and fixed with $4 \%$ formaldehyde. Tumor and lung tissue extracts were analyzed by hematoxylin and eosin (H\&E) staining and immunohistochemical analysis.

Immunohistochemical analysis. Immunohistochemical analyses were performed as previously described. Briefly, all tissue samples were fixed in phosphate-buffered neutral formalin, embedded in paraffin and cut into $5-\mu \mathrm{m}$ serial sections. After being deparaffinized in xylene, tissue sections were rehydrated in graded ethanol solutions, permeabilized in $0.1 \%$ Triton $\mathrm{X}-100$ and $0.1 \%$ sodium citrate and incubated overnight with primary antibodies. Immunohistochemical staining with antibodies to E-cadherin (1:50), vimentin (1:25, both from BD Biosciences) and ACE2 (1:50, R\&D Systems) was performed according to standard procedures. Results were observed and photographed under a fluorescence microscope (Leica, Germany) with Image-Pro Plus 6.0 software (Media Cybernetics). Specimens were classified as positive 
A

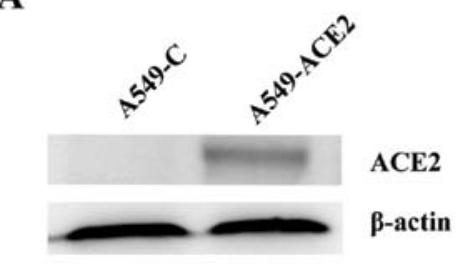

B

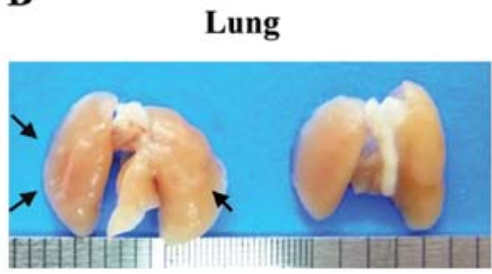

Liver

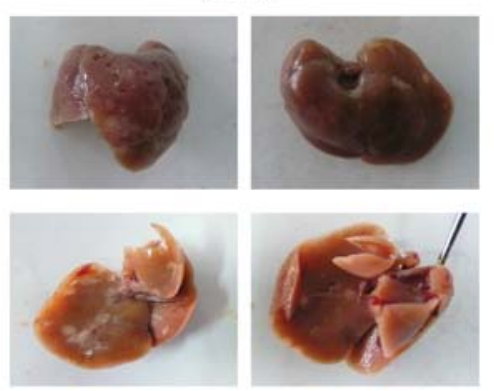

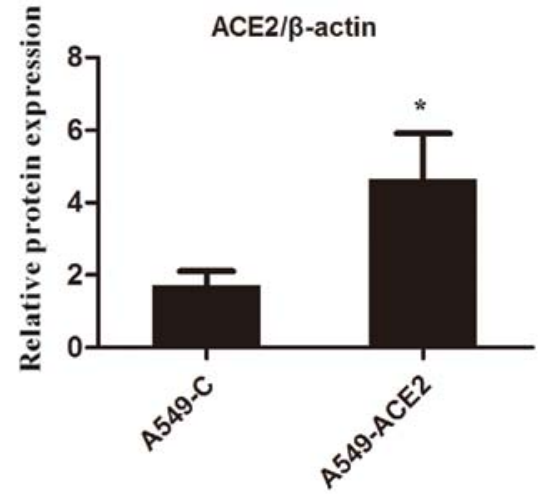

C

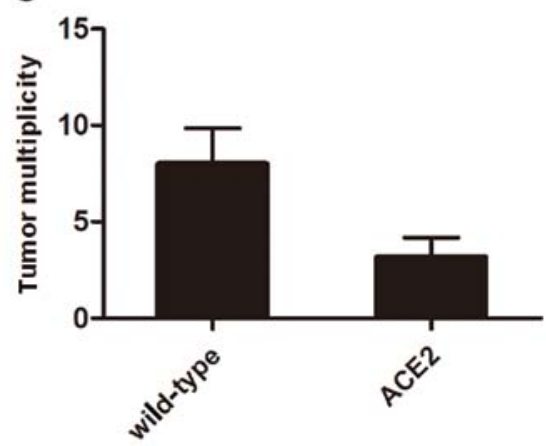

Figure 1. Effect of ACE2 on the metastasis formation of A549 cells. ACE2 suppressed the metastasis of A549 lung cancer cells in vivo. (A) A549 cells were injected in mice as described in Materials and methods. Overexpression of ACE2 in A549 cells (A549-ACE2) was confirmed by western blot analysis compared to wild-type cells (A549-C). Experiments were carried out in triplicate and representative bands are shown. Data represent the means \pm SEM of three independent experiments; ${ }^{*} \mathrm{P}<0.05$ when compared to A549-C cells. (B) Photomicrographs of representative lung and liver tumors extracted from nu/nu mice. Six-week-old male nu/nu mice were injected with A549-C or A549-ACE2 cells through vena caudalis. After three weeks, mice were sacrificed, and the lung, liver and brain tissues were extracted. Arrows indicate visible metastatic lesions as determined by gross examination of lung and liver tissues. Left panels indicate tissues extracted form the A549-C group; right panels indicate tissues extracted from the A549-ACE2 group (top panel, lung tissue; middle panel, abdominal aspect of liver; bottom panel, dorsal aspect of liver). (C) Tissue extracts were analyzed by hematoxylin and eosin (H\&E) staining to count the metastatic lesions. Histologically determined metastatic lesions were counted in the lung, liver and brain of mice injected with wild-type and A549-ACE2-cells. Data represent the means $\pm \mathrm{SD}$ ( $\mathrm{n}=6$ /group).

when $>10 \%$ of cancer cells were stained. The intensity of each type of staining was graded as negative or positive microscopically as previously reported (12).

Statistical analysis. Data are presented as averages and their respective standard deviation (means \pm SEM). All statistical analyses were performed with the SPSS Statistical Program (version 17.0; SPSS, Chicago, IL, USA). Comparisons of data between two groups were conducted with the Student's t-test. P-values of $<0.05$ were considered to indicate statistically significant results.

\section{Results}

Overexpression of ACE2 decreases metastasis of lung cancer in a mouse model. Our previous study showed that the overexpression of ACE2 inhibited the proliferation of lung cancer cells in vitro. It was also demonstrated that ACE2 overexpression reduced tumor growth in a mouse lung xenograft model (10). However, it is still unknown whether ACE2 decreases metastasis formation of lung cancer. Consequently, a cell line stably overexpressing ACE2 (A549-ACE2) was constructed in the present study. As shown in Fig. 1A, the increased expression of ACE2 in A549-ACE2 cells was confirmed by western blot analysis compared to the barely detectable levels in the wild-type cells (A549-C). In our model of lung cancer metastasis, gross examination of the lungs and livers extracted from nu/nu mice revealed numerous tumors (Fig. 1B). Age-matched nu/nu mice transplanted with A549 cells overexpressing ACE2 developed significantly fewer tumors $(\mathrm{P}<0.05)$ (Fig. 1C). These data revealed that the overexpression of ACE2 reduced the metastatic potential of lung cancer cells in vivo. Therefore, the regulation of ACE2 expression could prove a novel strategy for anticancer therapy.

Overexpression of ACE2 upregulates expression of E-cadherin in vitro. EMT has been recently found to play a critical role in tumor development, particularly during the induction of metastasis (14). Decrease or loss of E-cadherin expression is a key event during EMT. We previously showed that ACE2 overexpression inhibited lung cancer metastasis in vivo, therefore we hypothesized that ACE2 decreases lung cancer metastasis 
A

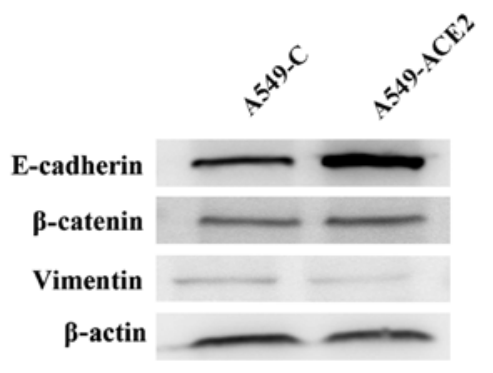

B

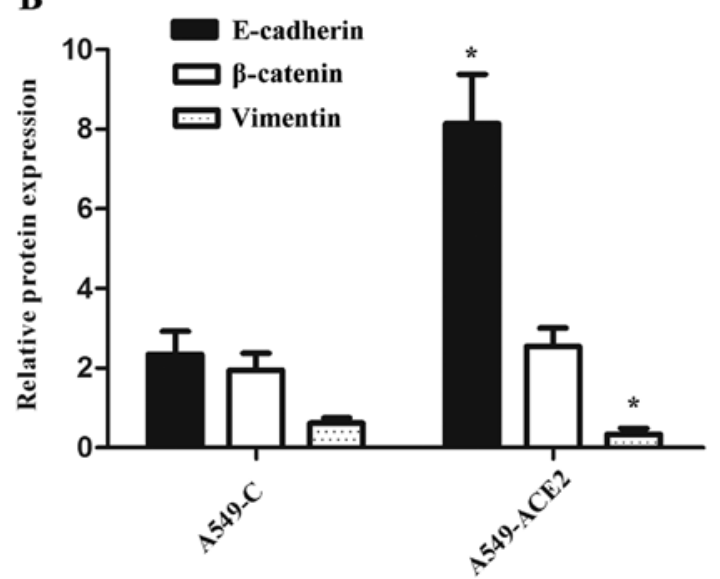

Figure 2. Effects of ACE2 overexpression on epithelial (E-cadherin and $\beta$-catenin) and mesenchymal (vimentin) markers in A549 cells in vitro. (A) E-cadherin, $\beta$-catenin and vimentin expression was determined in A549-C and A549-ACE2 cells using western blotting (whole cell lysate). $\beta$-actin was used as an internal control of protein loading. Overexpression of ACE2 increased E-cadherin expression and reduced vimentin in A549 lung cancer cells. Data are representative of three independent experiments. (B) Protein levels were quantified with ImageJ software and compared to $\beta$-actin as a loading control. ${ }^{*} \mathrm{P}<0.05$ when compared to A549-C cells.

through inhibiting EMT. To test this hypothesis, we utilized the previous cell lines to study the role of ACE2 on the expression of E-cadherin in vitro. To examine the influence of ACE2 on epithelial markers, including E-cadherin and $\beta$-catenin, we performed western blot analysis using anti-E-cadherin and anti- $\beta$-catenin antibodies (Fig. 2A). ACE2 overexpression significantly increased the expression of E-cadherin when compared to wild-type cells. This was determined by densitometric analysis of the western blot bands (Fig. 2B). In contrast, mesenchymal markers, particularly vimentin, were decreased in A549-ACE2 cells when compared to A549-C cells.

ACE2 influences the expression of EMT markers in a lung cancer xenograft model. We then evaluated the doubling times of A549-C and A549-ACE2 cells in vivo. A549-C or A549-ACE2 cells $\left(2 \times 10^{7}\right)$ were injected subcutaneously in mice; 21 days later, the resulting tumors were extracted. Immunohistochemical staining showed that the expression of E-cadherin was significantly higher and was located mainly on the cell membrane at the cell-cell junctions in all tumor specimens of the A549-ACE2 mouse group (Fig. 3C). Similarly, we found that ACE2 was localized on the cell membrane of A549 cells overexpressing ACE2 (4/6 positive in the A549-ACE2 group vs. $2 / 6$ positive in the control group). In addition, the patterns of expression and distribution of mesenchymal
A

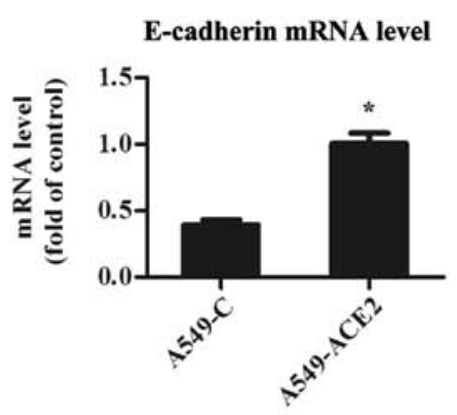

B

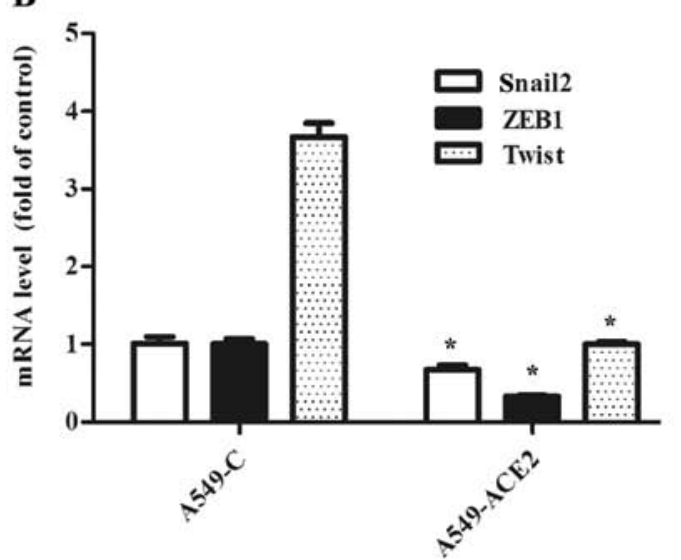

C
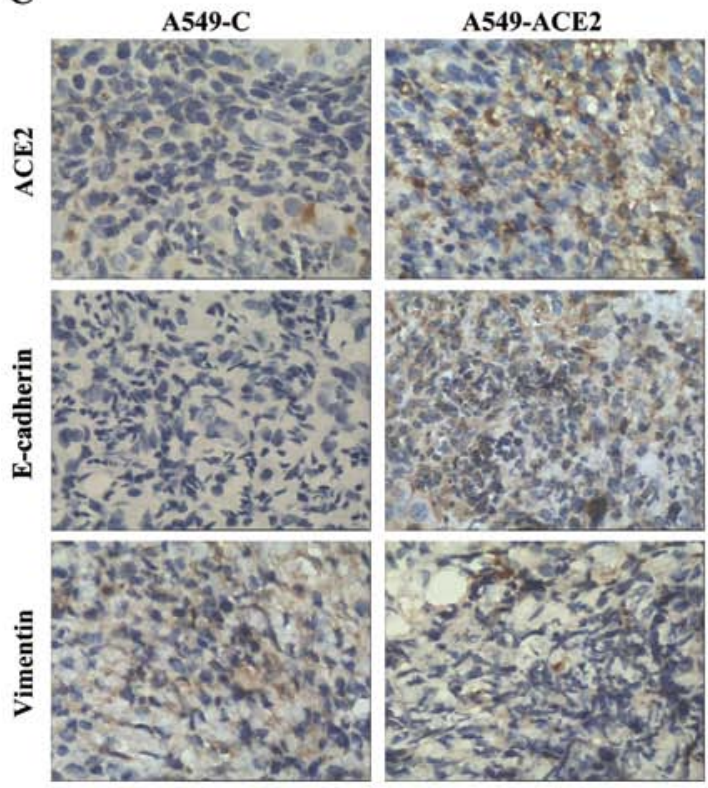

Figure 3. Effect of ACE2 on the transcription levels of EMT markers in a lung cancer xenograft model. (A) E-cadherin mRNA levels in tumor tissues of a mice xenograft model; ${ }^{\mathrm{P}}<0.01$ when compared to the A549-C group. (B) RT-PCR results of EMT-associated transcription. mRNA levels of Snail2, ZEB1 and Twist were significantly lower in the A549-ACE2 mouse group when compared to the A549-C group ( $\mathrm{P}<0.01)$. (C) Expression analysis of ACE2, E-cadherin and vimentin by immunohistochemistry in nu/nu mice was performed, and representative images of primary tumor sections are shown. Top panel, immunohistochemistry detecting ACE2 in subcutaneous tumors of nu/nu mice transplanted with A549 cells overexpressing ACE2 (A549-ACE2) or control A549 (A549-C) cells; middle panel, immunohistochemistry detecting E-cadherin; bottom panel, immunohistochemistry detecting vimentin. Original magnification, $x 400$.

markers were different between the two groups. Notably, the transfection of A549 cells with ACE2 led to a decreased vimentin expression (Fig. 4). Vimentin expression has been 
A
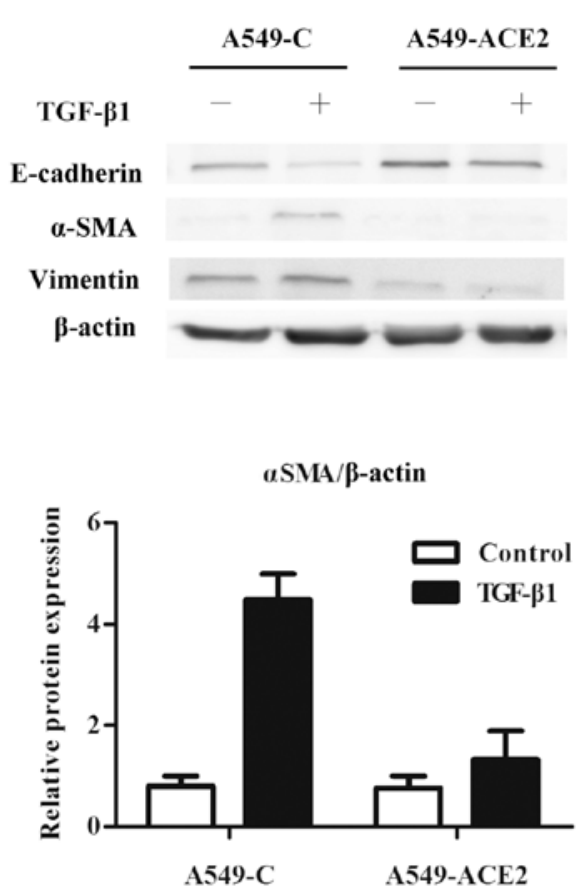

B

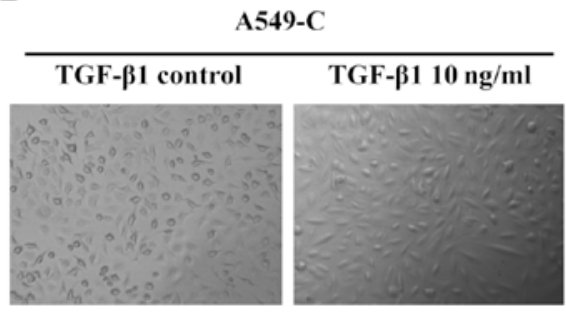

E-cadherin/ $\beta$-actin

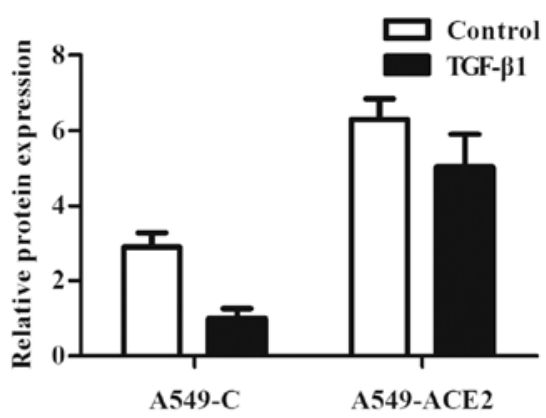

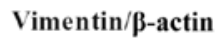

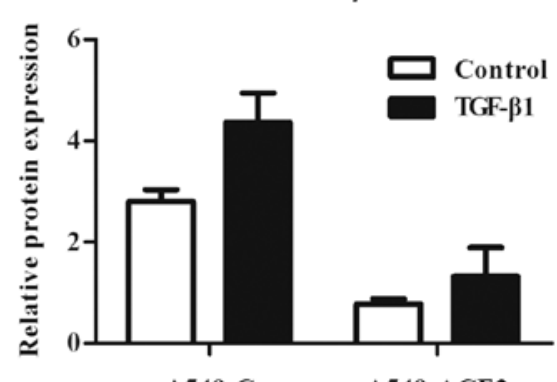

A549-C

A549-ACE2

Figure 4. ACE2 overexpression inhibits EMT in A549 cells. (A) Western blot analysis of EMT markers in control (A549-C) and ACE2-overexpressing cell lysates (A459-ACE2). Cells were treated for 3 days with or without $10 \mathrm{ng} / \mathrm{ml}$ TGF- $\beta 1$. The experiments were repeated three times and a representative blot is shown. The blot was stripped and re-probed with the $\beta$-actin antibody to detect the total amount of the respective proteins. Protein band intensities were quantified by densitometric analysis using the ImageJ software (National Institutes of Health). Columns, mean of triplicate experiments; bars, SE; "P<0.05. (B) Cell morphologic phenotypes of A549-C and A549-ACE2 cells were examined using a phase contrast microscope. TGF- $\beta 1$-treated A549-C cells showed a spindle-like shape and a loss of cell-to-cell attachments. Untreated A549-C and A549-ACE2 cells with or without TGF- $\beta 1$ treatment retained the morphological appearance of epithelial cells.

shown to positively correlate with EMT. In the A549-ACE2 mouse group, vimentin was expressed in only one specimen while it was expressed in 4 specimens extracted from the control group. In addition, we found that overexpression of ACE2 in A549 cells significantly upregulated the expression of epithelial-specific genes such as E-cadherin; E-cadherin is associated with decreased expression of mesenchymal markers (Fig. 3A).

ACE2 decreases mRNA levels of transcriptional repressors which are associated with EMT. We aimed to ascertain whether A549 cells overexpressing ACE2 had a differential expression pattern of genes involved in the induction of EMT. ACE2 overexpression was correlated with decreased mRNA levels of transcripts such as Snail2, ZEB1 and Twist, which are causally involved in the EMT process (Fig. 3B). In contrast, the mRNA level of E-cadherin was restored in the tumor tissues of the A549-ACE2 mouse group compared to the A549-C group (Fig. 3A). These data are consistent with the notion that ACE2 inhibits a set of genes and markers involved in EMT in a lung cancer xenograft model.

ACE2 attenuates EMT of A549 cells induced by TGF- $\beta 1$ in vitro. The upregulatory effect of ACE2 on E-cadherin expression in lung cancer cells led us to explore the direct impact of ACE2 on EMT. To further investigate whether ACE2 suppresses the EMT process, a classical EMT model was developed using A549 cells stimulated with TGF- $\beta 1$. The presence of ACE2 in A549 cells attenuated the decrease in the levels of E-cadherin due to TGF- $\beta 1$ treatment. In addition, ACE2 significantly abrogated the upregulation of mesenchymal markers, including vimentin and $\alpha$ SMA (Fig. 4). The cell morphologic phenotypes of A549-C and A549-ACE2 cells were examined using a phase contrast microscope. TGF- $\beta 1$-treated A549-C cells showed a spindle-like shape and a loss of cell-to-cell attachments. Untreated A549-C and A549-ACE2 cells with or without TGF- $\beta 1$ treatment retained the morphological appearance of epithelial cells (Fig. 4B). 
A
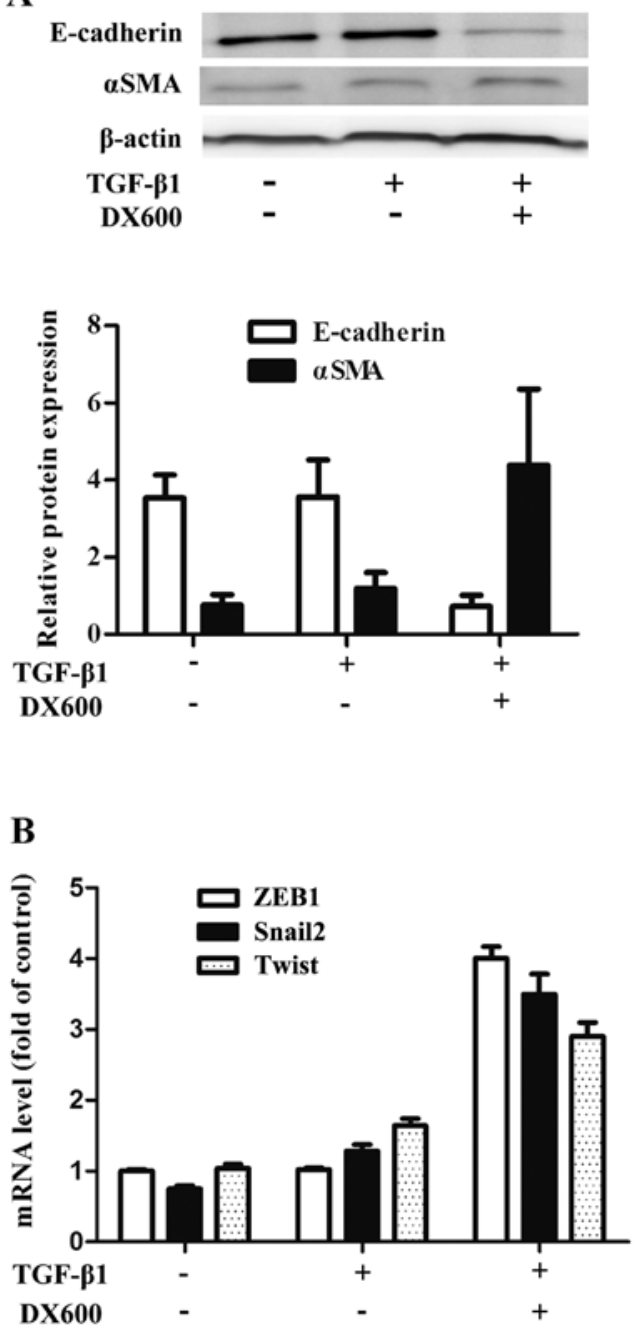

Figure 5. Effect of DX600 on the expression of EMT markers. (A) Western blot analysis of the epithelial marker E-cadherin and the mesenchymal marker $\alpha$ SMA was carried out in A549-ACE2 cells with or without TGF- $\beta 1$ or DX600 treatment. Mean percentage values were obtained from the results of three independent experiments. (B) Transcription levels of Snail2, ZEB1 and Twist in A549-ACE2 cells treated either with TGF- $\beta 1$ alone or in combination with DX600 using RT-PCR. The relative expression of mRNAs was normalized to that of the endogenous control ( $\beta$-actin). All the experiments were performed in triplicate. Data represent the means \pm SEM.

Effect of ACE2 inhibition on the expression of EMT markers. An ACE2 antagonist was used to further confirm the effect of ACE2 on EMT. Pretreatment of A549 cells with DX600 (at $10^{6} \mathrm{M}$ ) abolished the increase in E-cadherin expression caused by ACE2. Furthermore, the upregulation of $\alpha$ SMA caused by TGF- $\beta 1$ treatment was recovered in A549-ACE2 cells following treatment with DX600 (Fig. 5A). A549-ACE2 cells treated with TGF $\beta-1$ followed by treatment with DX600, lost the effect of ACE2-mediated restoration of E-cadherin expression to control levels. These results demonstrate that the lack of ACE2 increases the sensitivity of A549 cells to TGF- $\beta 1$-induced EMT.

In order to mechanistically investigate the role of ACE2 in the regulation of transcriptional repressors, including Snail2 and ZEB1 during the process of EMT, we determined the transcriptional repertoire of A549 cells following treatment with or without TGF- $\beta 1$. We found that the expression levels of ZEB1, Snail2 and Twist were markedly higher in A549-ACE2 cells treated with TGF- $\beta 1$ and DX600 when compared to TGF- $\beta 1$ treatment alone. This was consistent with the increased expression of mesenchymal markers (Fig. 5B).

\section{Discussion}

In the present study we investigated the role of ACE2 in lung cancer metastasis and EMT. To the best of our knowledge, this is the first demonstration of how ACE2, in a A549 cell lung cancer model, decreases metastasis in vivo. ACE2 upregulates the expression of E-cadherin both in vitro and in vivo as well as downregulates vimentin. These proteins are representative markers of the EMT process. Furthermore, western blot analysis indicated that ACE2 attenuated TGF- $\beta 1$-mediated EMT of A549 cells. ACE2 decreased the transcriptional levels of genes associated with EMT in vitro. Exposing cells to DX600, an inhibitor of ACE2, recovered the sensitivity of lung cancer cells to TGF- $\beta 1$.

Components of the RAS system have been found to influence tumor growth and development (15-17). ACE2 is a critical enzyme of the RAS system, which plays a counterbalancing role by degrading Ang II to Ang 1-7 (18). Although numerous large clinical trials have been carried out, the contribution of the RAS system to tumor development remains controversial $(19,20)$. One study showed a negative association of losartan and a positive association of candesartan and telmisartan with the overall occurrence of cancer (21). Despite the different origins of cancer, the results still remain distinct. Bhaskaran et al (22) found a decreased risk of lung cancer but increased risks for breast and prostate cancers. The contradictory findings among the two methods of angiotensin receptor blockade (ARB) suggest that a more complicated regulatory mechanism exists in the RAS system. As the RAS system is not linear, we hypothesized that the imbalance of the different pathways of RAS plays a critical role in tumor growth and development, and not Ang II and its receptors alone. Among these, ACE2 may be the key component to link and regulate opposing Ang II and Ang 1-7 pathways (23). Several components of RAS may be expressed in the lung (24). The local concentration of these cytokines is also altered in different lung diseases. The beneficial effect of ACE2 has been studied in pulmonary hypertension and pulmonary fibrosis (25).

In lung cancer, we found that decreased expression of ACE2 correlates with poor clinical outcomes, which suggested that ACE2 acts as a suppressor of lung cancer progression. It has also been reported that the overexpression of ACE2 in human pancreatic carcinoma cells decreased tumor growth, both in vitro and in vivo. To examine the influence of ACE2 on lung cancer metastasis, we evaluated in two xenograft experiments the doubling times of A549-C and A549-ACE2 cancer cells. Here, ACE2 significantly attenuated the metastasis of lung cancer in vivo.

Several key factors have been noted to be involved in the malignant behavior of cancer cells. For example, loss of E-cadherin has been considered an early event in cancer development, which is well known to promote cancer cell invasion and lymph node metastasis. Recently, EMT has been reported to play a role in tumor progression $(14,26)$. The results of our study showed that ACE2 overexpression markedly attenuated the effect of TGF- $\beta 1$-induced EMT. Notably, TGF- $\beta 1$ is a key mediator of the EMT process $(27,28)$. Ang II, 
which is known as a key molecule of the RAS system, shares many cellular responses with TGF- $\beta 1$ (29). In renal epithelial cells, treatment with Ang II resulted in a transition of cellular morphology from a 'cobblestone' epithelial to a mesenchymal phenotype. Altered expression of the biomarkers for EMT were also demonstrated (30). Ang II-induced key events of the EMT process in intrahepatic cholangiocarcinoma were found to include the downregulation of epithelial adherins and upregulation of vimentin. Ang II was found to enhance cell invasiveness and migration. Ang II may also serve as a growth factor in tumor development and facilitate tumor metastasis of cancer cells (31). However, it is still unknown whether Ang II is a stimulus of EMT in lung cancer.

In the present study, we found that ACE2, which is a newly identified component of the RAS system, attenuated lung cancer metastasis through its inhibition of the EMT process. This finding suggests that ACE2 may be a potential therapeutic target of lung cancer where EMT contributes to the development of tumor metastasis.

\section{Acknowledgements}

This study was supported by a grant from the National Natural Science Foundation of China (81071925).

\section{References}

1. Siegel R, Naishadham D and Jemal A: Cancer statistics, 2012. CA Cancer J Clin 62: 10-29, 2012.

2. Thiery JP, Acloque H, Huang RY and Nieto MA: Epithelialmesenchymal transitions in development and disease. Cell 139: 871-890, 2009

3. Zhou L, Zhang R, Zhang L, Yao W, Li J and Yuan Y: Angiotensinconverting enzyme 2 acts as a potential molecular target for pancreatic cancer therapy. Cancer Lett 307: 18-25, 2011.

4. Pickel L, Matsuzuka T, Doi C, Ayuzawa R, Maurya DK, Xie S-X Berkland $\mathrm{C}$ and Tamura M: Overexpression of angiotensin II type 2 receptor gene induces cell death in lung adenocarcinoma cells. Cancer Biol Ther 9: 277-285, 2010.

5. George AJ, Thomas WG and Hannan RD: The renin-angiotensin system and cancer: old dog, new tricks. Nat Rev Cancer 10 745-759, 2010.

6. Wilop S, von Hobe S, Crysandt M, Esser A, Osieka R and Jost E: Impact of angiotensin I converting enzyme inhibitors and angiotensin II type 1 receptor blockers on survival in patients with advanced non-small-cell lung cancer undergoing first-line platinum-based chemotherapy. J Cancer Res Clin Oncol 135: 1429-1435, 2009.

7. LiH, Qi Y, Li C, Braseth LN, Gao Y, Shabashvili AE, Katovich MJ and Sumners C: Angiotensin type 2 receptor-mediated apoptosis of human prostate cancer cells. Mol Cancer Ther 8: 3255-3265, 2009.

8. Redondo-Müller MA, Stevanovic-Walker M, Barker S, Puddefoot JR and Vinson GP: Anti-cancer actions of a recombinant antibody (R6313/G2) against the angiotensin II AT1 receptor. Endocr Relat Cancer 15: 277-288, 2008.

9. Nakai Y, Isayama H, Ijichi H, Sasaki T, Sasahira N, Hirano K, Kogure H, Kawakubo K, Yagioka H, Yashima Y, et al: Inhibition of renin-angiotensin system affects prognosis of advanced pancreatic cancer receiving gemcitabine. Br J Cancer 103: 1644-1648, 2010.

10. Feng Y, Ni L, Wan H, Fan L, Fei X, Ma Q, Gao B, Xiang Y, Che J and Li Q: Overexpression of ACE2 produces antitumor effects via inhibition of angiogenesis and tumor cell invasion in vivo and in vitro. Oncol Rep 26: 1157-1164, 2011.

11. Okamoto K, Tajima H, Nakanuma S, Sakai S, Makino I, Kinoshita J, Hayashi H, Nakamura K, Oyama K, Nakagawara H, et al: Angiotensin II enhances epithelial-to-mesenchymal transition through the interaction between activated hepatic stellate cells and the stromal cell-derived factor-1/CXCR4 axis in intrahepatic cholangiocarcinoma. Int J Oncol 41: 573-582, 2012.
12. Feng Y, Wan H, Liu J, Zhang R, Ma Q, Han B, Xiang Y, Che J, Cao H, Fei X, et al: The angiotensin-converting enzyme 2 in tumor growth and tumor-associated angiogenesis in non-small cell lung cancer. Oncol Rep 23: 941-948, 2010.

13. Ni L, Feng Y, Wan H, Ma Q, Fan L, Qian Y, Li Q, Xiang Y and Gao B: Angiotensin-(1-7) inhibits the migration and invasion of A549 human lung adenocarcinoma cells through inactivation of the PI3K/Akt and MAPK signaling pathways. Oncol Rep 27: 783-790, 2012.

14. Iwatsuki M, Mimori K, Yokobori T, Ishi H, Beppu T, Nakamori S, Baba $\mathrm{H}$ and Mori M: Epithelial-mesenchymal transition in cancer development and its clinical significance. Cancer Sci 101: 293-299, 2010

15. Puddefoot JR, Udeozo UK, Barker S and Vinson GP: The role of angiotensin II in the regulation of breast cancer cell adhesion and invasion. Endocr Relat Cancer 13: 895-903, 2006.

16. Uemura $\mathrm{H}$, Ishiguro $\mathrm{H}$, Nagashima $\mathrm{Y}$, Sasaki T, Nakaigawa $\mathrm{N}$, Hasumi H, Kato S and Kubota Y: Antiproliferative activity of angiotensin II receptor blocker through cross-talk between stromal and epithelial prostate cancer cells. Mol Cancer Ther 4: 1699-1709, 2005.

17. Kanehira T, Tani T, Takagi T, Nakano Y, Howard EF and Tamura M: Angiotensin II type 2 receptor gene deficiency attenuates susceptibility to tobacco-specific nitrosamine-induced lung tumorigenesis: involvement of transforming growth factor-betadependent cell growth attenuation. Cancer Res 65: 7660-7665, 2005.

18. Tikellis C, Bernardi S and Burns WC: Angiotensin-converting enzyme 2 is a key modulator of the renin-angiotensin system in cardiovascular and renal disease. Curr Opin Nephrol Hypertens 20: 62-68, 2011.

19. Bangalore S, Kumar S, Kjeldsen SE, Makani H, Grossman E, Wetterslev J, Gupta AK, Sever PS, Gluud C and Messerli FH: Antihypertensive drugs and risk of cancer: network meta-analyses and trial sequential analyses of 324,168 participants from randomised trials. Lancet Oncol 12: 65-82, 2011.

20. Pasternak B, Svanström H, Callréus T, Melbye M and Hviid A: Use of angiotensin receptor blockers and the risk of cancer. Circulation 123: 1729-1736, 2011.

21. Chang CH, Lin JW, Wu LC and Lai MS: Angiotensin receptor blockade and risk of cancer in type 2 diabetes mellitus: a nationwide case-control study. J Clin Oncol 29: 3001-3007, 2011.

22. Bhaskaran K, Douglas I, Evans S, van Staa T and Smeeth L: Angiotensin receptor blockers and risk of cancer: cohort study among people receiving antihypertensive drugs in UK General Practice Research Database. BMJ 344: e2697, 2012.

23. Ferrario CM: ACE2: more of Ang-(1-7) or less Ang II? Curr Opin Nephrol Hypertens 20: 1-6, 2011.

24. Gembardt F, Sterner-Kock A, Imboden H, Spalteholz M, Reibitz F, Schultheiss HP, Siems WE and Walther T: Organspecific distribution of ACE2 mRNA and correlating peptidase activity in rodents. Peptides 26: 1270-1277, 2005.

25. Shenoy V, Ferreira AJ, Qi Y, Fraga-Silva RA, Diez-Freire C, Dooies A, Jun JY, Sriramula S, Mariappan N, Pourang D, et al: The angiotensin-converting enzyme 2/angiogenesis-(1-7)/Mas axis confers cardiopulmonary protection against lung fibrosis and pulmonary hypertension. Am J Respir Crit Care Med 182: 1065-1072, 2010.

26. Hanahan D and Weinberg RA: Hallmarks of cancer: the next generation. Cell 144: 646-674, 2011.

27. Moustakas A and Heldin CH: Induction of epithelial-mesenchymal transition by transforming growth factor $\beta$. Semin Cancer Biol 22: 446-454, 2012.

28. Perez RE, Navarro A, Rezaiekhaligh MH, Mabry SM and Ekekezie II: TRIP-1 regulates TGF- $\beta 1$-induced epithelialmesenchymal transition of human lung epithelial cell line A549. Am J Physiol Lung Cell Mol Physiol 300: L799-L807, 2011.

29. Arnold SA, Rivera LB, Carbon JG, Toombs JE, Chang CL, Bradshaw AD and Brekken RA: Losartan slows pancreatic tumor progression and extends survival of SPARC-null mice by abrogating aberrant TGF $\beta$ activation. PLoS One 7: e31384, 2012.

30. Burns WC, Velkoska E, Dean R, Burrell LM and Thomas MC: Angiotensin II mediates epithelial-to-mesenchymal transformation in tubular cells by ANG 1-7/MAS-1-dependent pathways. Am J Physiol Renal Physiol 299: F585-F593, 2010.

31. Rodrigues-Ferreira S, Abdelkarim M, Dillenburg-Pilla $P$, Luissint AC, di-Tommaso A, Deshayes F, Pontes CL, Molina A, Cagnard N, Letourneur F, et al: Angiotensin II facilitates breast cancer cell migration and metastasis. PLoS One 7: e35667, 2012. 